\title{
The Biophysical Modeling of Fracture Types
}

\author{
Janos Vincze, Gabriella Vincze-Tiszay \\ Health Human International Environment Foundation, Budapest, Hungary
}

Email address:

ndp@t-online.hu (J. Vincze)

\section{To cite this article:}

Janos Vincze, Gabriella Vincze-Tiszay. The Biophysical Modeling of Fracture Types. American Journal of Internal Medicine. Vol. 8, No. 1, 2020, pp. 34-39. doi: 10.11648/j.ajim.20200801.17

Received: January 21, 2020; Accepted: February 10, 2020; Published: February 18, 2020

\begin{abstract}
The fracture leads immediately to the impossibility to perform certain movements, followed by tumefaction, sharp pain, haematoma, sometimes a visible deformation and even a wound of the skin, in the case of an open fracture. For any person who has the signs of fracture, that region must be immobilized immediately during first aid and for the final healing they must be taken to the hospital. A fracture can appear in various circumstances: through a direct- and indirect-mechanism or is called "pathological bone fracture". The degree of fracture we distinguish: incomplete fractures, complete fractures and avulsions. The knowledge of fracture types has therapeutic implications. The very frequently fracture types are next: compression, rupture, bending, extension, torsion. In this paper we use the biophysical modeling of different fracture types. To get hold of the biophysical view is not an easy task, because it applies mathematical apparatus to biological systems, on the other hand as a reward it guides to fascinating results, recognizing theoretically which conformity of rules are valid on principle in the inanimate-living-spiritual triple system from the lowest to the highest organizational level.
\end{abstract}

Keywords: Degree of Fracture, Biophysical Modeling, Fracture Types, Restoring Fitness

\section{Introduction}

The break in the continuity of the bone is called fracture (fractura). The exact description should include the position of the broken ends (proximalis, distalis), the course of the fracture gap, the presence of any broken bone fragments and the remains zone, the type and extent of displacement. [1]

Among the external factors, the decisive factors are the extent, duration, direction and rate of growth of the force trauma. When a solid body is subjected to an ever increasing force, it undergoes a dimensional change as a result of the generated stress. [2] The initially flexible elongation regains its original size once the stress stops, then deforms beyond the flexible limit and is interrupted (fractured) by further stress.

Among the internal factors, the energy-absorbing capacity of the bone, its coefficient of flexibility, its density and its resistance to stress (cyclic) forces are important. [3] The bone is a biphasic material consisting mainly of a collagen matrix and a mineral part (predominantly hydroxyapatite). Collagen provides resistance to elongation, while the mineral component provides stiffness and resistance to compression. [4] The special arrangement of the apatite crystals has an energy absorbing effect and the static solidity is exponentially proportional to the degree of mineralization.

In order to know the types of fractures, it is important to know the physical characteristics of the bones (see in the Table 1) [5].

Table 1. The physical characteristics of the bones.

\begin{tabular}{ll}
\hline Parameters & Values \\
\hline Density & $1,92-1,99 \mathrm{~g} / \mathrm{cm}^{3}$ \\
Elasticity module & $16-27.10^{9} \mathrm{~N} / \mathrm{m}^{2}$ \\
Breaking coefficient & $9,1-12,4 \mathrm{~kg} / \mathrm{mm}^{2}$ \\
Compression coefficient & $12,1-17 \mathrm{~kg} / \mathrm{mm}^{2}$ \\
Shearing coefficient & \\
$\quad$ in longitudinal direction & $5,0 \mathrm{~kg} / \mathrm{mm}^{2}$ \\
$\quad$ in transversal direction & $12,8 \mathrm{~kg} / \mathrm{mm}^{2}$ \\
Torsion coefficient & $4,0-9,3 \mathrm{~kg} / \mathrm{mm}^{2}$ \\
\hline
\end{tabular}

Due to its anisotropic structure, the coefficient of flexibility of the bone is significantly higher at longitudinal tension than at any other tension (which explains the experience that much less force is enough for a fracture when it is twisted than in the longitudinal or transverse direction). In the area of bone holes, bone is weakened and the critical limit is when the diameter of the bone hole exceeds one third of the bone diameter. [6] 


\section{The Diagnosis of Fracture}

The diagnosis is supported by sight, tactile evidence $\mathrm{x}$-ray and possibly ultrasound [7]. In the case of anamnesis, primarily, data on the present injury are collected in the medical history. We are often forced to perform a heteroanamnesis when the injured person is unconscious or otherwise unable to provide the necessary information. Regarding the present injury, both a subjective and objective (relatives or paramedics) medical history should be obtained. Thus, the time, place and exact circumstances of the injury should be recorded. One should strive to find out the amount of energy that was involved in the injury, because thus the extent of the trauma can be inferred. Clarifying the state of the injured at the time of the accident helps to identify injuries that are not apparent at first or covered secondary injuries. A comparison of objective and subjective medical history helps to identify the brain injury of a patient who is already unconscious at the time of examination. We gather information about the patient's pain and its location. Patients usually only report complaints resulting from the main injury, so that secondary injuries remain unrecognized. Thorough examination is therefore required. Knowledge of certain injury combinations is essential for a correct diagnosis. [8]

When examining injuries, we use a protractor to measure joint movements. The result may be given in absolute terms or as the extent of the deficit relative to the normal reference data, but more preferably to the healthy opposite.

The knowledge of fracture types has therapeutic implications. It helps in planning restabilization, determining stability, consequently in deciding which stabilization to choose, as well as in recognizing secondary injuries. If we see some larger fragments in the fracture zone, it is called a segmental fracture, if we have a zone with many smaller pieces that result in defect, it is called a comminuted fracture.

It is our job to achieve safe recovery and restore full function as soon as possible [9]. The steps are the following: repositio (repositioning), retentio (retention of the position reached) and rehabilitation. [10] The principles of repair: longitudinal pulling, reconstruction of the mechanism of injury in the reverse order and the repositioning of the movable fractured end to the non-movable end accordingly. Retention means fixing the achieved repository position until bone consolidation. This can be done by conservative or surgical methods [11].

Broken ends usually move. If this does not occur, it may be the best case. The fractured ends may move relative to one another: laterally, longitudinally (by shortening or recoiling), with axis misalignment and twisted rotational deviation. The different types of movement are usually combined. Movements of different sizes may be tolerated in each region, which will not interfere with the good function. Rotational dislocation is the least tolerated.

\section{The Degree of Fracture}

According to the degree of fracture we distinguish: incomplete fractures, complete fractures and avulsions.

An incomplete fracture is when the continuity, the interruption does not extend to the entire thickness of the bone. It has two characteristic forms: greenstick and fissure. In the case of greenstick fractures the bone cortex may be squeezed or the spongy bone may be compressed. Greenstick fracture occur on soft, flexible bones. Children's bone fractures are all greenstick fractures; in the case of adults, they can occur on the clavicle, the ribs, the sternum; on the atrophied bones of the elderly on the femoral neck for example. Impression may occur as a result of compression on the flat bones of the skull, the vertebral bodies, the spongy tissue of the tip of the long tubular bone, and the carpal bone and the tarsus. A fissure is the interruption of bone continuity without altering the shape of the bone. [12] It occurs on long tubular bones and flat bones.

The complete fracture is defined as a discontinuity through the full thickness of the bone. In this case, the periosteum also ruptures. The fractures are shifted by force or by the pulling of the muscles. With avulsions, bone discontinuity occurs at border of the joint end and the cartilage. It is very common in young people. [13]

The fracture can be closed, compound or complicated. In the case of a closed fracture, the skin above the bone continuity interruption is intact. In the case of a compound fracture, the fractured bone or the impact force will cause an interruption of continuity on the skin and the soft parts surrounding the fracture. The fractured parts are exposed. They can be easily infected. Complicated fracture is defined as bone discontinuity associated with damage to a joint, blood vessel, nerve, or internal organ (brain, lung, heart, bowel, bladder, etc.).

The injury needs immediate surgery, which must be done within 6 hours. Take down the dressing only under surgical conditions and do not use a tourniquet. The principle of treating second and third degree compound fractures is thorough mechanical cleaning and excision, maximally stable bone fixation, providing a viable, high blood supply soft tissue mantle, covering the bone and joints, and treating the wound itself.

\section{The Types of Fractures}

The most common types of fractures are:

1) Direct trauma [14]: a fracture occurs as a direct result of force trauma and in the same place.

a) Defensive fracture, such as the fracture of the forearm in the defense of a blow from a stick. Usually only one bone breaks transversely.

b) Compression fracture, where severe soft tissue injury can always be expected. In the lower leg and the forearm both bones break transversely, at the same height, frequently accompanied by an extensive fracture zone.

In the case of compression fracture [15] two contrary forces act on the epiphyses along the axis. The tension $\sigma$ which appears in the bone is calculated on the basis of the 
relation

$$
\sigma=\text { H. } \varepsilon
$$

where: $\mathrm{H}$ - bone compression coefficient; $\varepsilon$ - deformation tensor. In the case of compression the deformation tensor is ellipsoid, whose coordinates satisfy the following relation:

$$
\varepsilon_{\mathrm{xx}} \cdot \mathrm{x}^{2}+\varepsilon_{\mathrm{yy}} \cdot \mathrm{y}^{2}+\varepsilon_{\mathrm{zz}} \cdot \mathrm{z}^{2}+2 \cdot \varepsilon_{\mathrm{xy}} \cdot \mathrm{xy}+2 \cdot \varepsilon_{\mathrm{xz}} \cdot \mathrm{xz}+2 \cdot \varepsilon_{\mathrm{yz}} \cdot \mathrm{yz}=\mathrm{k}
$$

where: $\mathrm{k}$ - a constant; $\varepsilon_{\mathrm{xx}}, \varepsilon_{\mathrm{yy}}, \varepsilon_{\mathrm{zz}}, \varepsilon_{\mathrm{xy}}, \varepsilon_{\mathrm{xz}}, \varepsilon_{\mathrm{yz}}-$ are the coefficient of the deformation tensor.

c) Penetrating (gunshot) fracture. These are divided into two groups according to the speed of the projectile. According to this: $1 . /$ below $600 \mathrm{~m} / \mathrm{sec}$, damage is caused by a low speed (low energy) projectile, and $2 . /$ above $600 \mathrm{~m} / \mathrm{s}$, damage is caused by a high speed (high energy) projectile. The latter are mostly caused by military weapons. There is a significant difference between the two, because in the former, only the bone ruptures, or the bullet gets stuck in the spongiosus bone, and there is only a small amount of soft tissue damage, while the latter causes an extensive bone and soft tissue destruction.

A torus fracture is created by two transverse forces acting against the bone, their points of attack facing each other, or by a force acting on a fixed bone at the edge of the support. When the force is high and fast, it only pushes the bone parts in the direction of pressure at the point of impact. In this case, the fracture is transverse. If the magnitude and velocity of the force is smaller, it will affect areas further away from the point of impact, whereby the bending force will prevail. The transverse fracture that starts transversely as the force exerts is slanting in the fixed direction.

In the case of torus fractures, the shear tangential stress is $(\tau): \tau=F / A$, where $F$ is the applied force and $A$ is the surface of the cut section.

Torus fracture is usually accompanied by bending, twisting and crushing. In general, piercing, cut and shot injuries to the skull are torus fractures. The broken bone fragment usually has the shape of the device that caused the injury. The fracture is influenced by the magnitude, direction and impulse of the impact force.

If the pulse of the projectile that causes the injury is small, a bend fracture will occur, if the momentum is high, a clear cut fracture will occur. The fracture also depends on the type of bone. A projectile with the same pulse punctures flat bones and pierces the cancellous bone of at the ends of long bones causing a typical torus fracture. On the other hand, in the case of long bones it causes comminuted fractures on the periosteum. The smaller the range of the projectile, the smaller the bone fragments.

2) Indirect trauma: when the fracture is caused by the impact of the force occurring at a distant point.

a) In the case of ruptures, a hyperextended tendon or ligament ruptures the bone. This is what happens on the patella, on the olecranon, when the knee or elbow bend under the high-tensioned extensor muscle. Similarly, the ankle can be ruptured by the deltoid ligament attached to it. The fracture line is transverse.

In the case of a rupture, a sudden pulling force separates a smaller piece of bone from the larger one (base). It can be caused by a strong, spasmodic contraction of the muscle at the site of its origin, adhesion, or ligament, when the penetrating force forces the joint to exaggerate physiological or abnormal movements. Usually, fractures of condyles and epicondyles are of this origin.

In the case of breaking fractures [16], several pieces of bones detach. In this case the force $F$ which acts in the point $\mathrm{P}(\mathrm{x} ; \mathrm{y})$ generates in point $\mathrm{Q}(\mathrm{u} ; \mathrm{v})$ the following breaking tension $\sigma$ :

$$
\sigma=\frac{F}{A} \cdot\left(1+\frac{u \cdot y}{i_{x}^{2}}+\frac{v \cdot x}{i_{y}^{2}}\right)
$$

where: $\mathrm{A}-$ is the bone transversal section; $\mathrm{i}_{\mathrm{x}}$ and $\mathrm{i}_{\mathrm{y}}$ are the rays of the invariable spinning moments, projected in the $\mathrm{x}$ and y directions.

Muscle tension may result in the tearing of the coronoid process of the mandible, the vertebrae processes, the scapula, greater and lesser tubercle of the humerus, the olecranon and inferior extremity of the ulna, the buttocks, the intercondyloid eminence of the tibia, the dorsal side of the calcaneus. Ligament tension may result in the tearing of the small condyle of the upper arm and thigh, the intercondylar tubercles of the tibia, the malleolus.

b) In the case of bend fractures, there is excess tension on the protuberance and compression on the concavity. The fracture line is roughly transversal, but on the concave side it is usually triangular, and a so-called bending wedge breaks out.

Bend fractures occur when two forces are applied perpendicular to the longitudinal axis of the bone, or a force is applied perpendicular to the longitudinal axis of the bone until the elastic limit and flexural strength of the bone is overcome. When we break a stick over our knees, the forces that apply to the stick touching our knee are clearly compression forces.

In the same place, tensile forces appear on the opposite side. Since solid bodies exhibit less strength towards pulling/tearing than towards compression, a transverse fracture on the opposite side of the force occurs. As the continuity interruption has penetrated to the center of the stick, the compressive and tensile forces are equalized and the fracture line continues in the longitudinal direction. When the fracture passes beyond the center line of the body, the fracture ends in a transverse direction due to pressure at the site of force application. If the overbent bone is struck at its maximum curvature, a triangular piece will bounce off the concavity.

If the points of attack of the transverse, opposing forces are far from each other, bend fracture will occur. Bending forces can cause simple cracks, greenstick fractures, oblique or traverse fractures. 
In the case of bending fractures [17], there is a force $F$ which acts on a long bone with the length $\mathrm{d}$, from the epiphyses of the bone at the distances $d_{1}$ and $d_{2}$, obviously $d$ $=\mathrm{d}_{1}+\mathrm{d}_{2}$ the forces $\mathrm{F}_{1}$ and $\mathrm{F}_{2}$ are calculated at the level of the epiphyses in the following manner:

$$
F_{1}=F \cdot \frac{d_{2}}{d} F_{2}=F \cdot \frac{d_{1}}{d}
$$

The maximum effect of the bending moment (M), respectively the inertia moment (I) after the action of the force $\mathrm{F}$, are given by the relations:

$$
M=F \cdot \frac{d_{1} \cdot d_{2}}{d} I=\frac{\pi \cdot r^{2}}{64}
$$

The values of the angles $\varphi_{1}$ and $\varphi_{2}$ at the end of the central area (the so called neutral area) is calculated on the basis of the following relation:

$$
\Delta d=\frac{1}{E} \cdot \frac{F}{A}
$$

where: E - bone elasticity module.

In the stretching fracture based on the force $\mathrm{F}$, for the bone with the diameter $\mathrm{A}$, a $\Delta \mathrm{d}$ stretching takes place:

$$
\begin{aligned}
& \operatorname{tg} \varphi_{1}=\frac{F \cdot d_{1} \cdot d_{2}\left(d_{1}+2 d_{2}\right)}{6 \cdot E \cdot I \cdot d} \\
& \operatorname{tg} \varphi_{2}=\frac{F \cdot d_{1} \cdot d_{2}\left(2 d_{1}+d_{2}\right)}{6 \cdot E \cdot I \cdot d}
\end{aligned}
$$

where: $\mathrm{E}$ - the bone elasticity module.

When the calva is hit from outside, tensile forces are applied to the inner plate, and this will break first. When the skull and the chest are squeezed by two forces from the opposite side, a bend fracture will occur at the highest point of the protuberance. When the force is applied transversely to a fixed bone at one end, the fracture line starts transverse and is inclined at an angle compared to the fixed portion That is how the bend and tension fractures of the upper arm occur. Compressive forces acting along the longitudinal axis also cause bend fractures in the natural curvatures of the bones: e.g. if a person falls from a height to his knees or to a lower leg with a stretched limb, he will suffer a bend fracture in his thigh.

Muscle tension can also cause fractures. For example, diving into the water can cause a fracture of the neck, or a strong cough may break the rib. The spasmodic contraction of the cephalic, thoracic and deltoid muscles may cause the rupture of the clavicle. A strong contraction of the deltoid muscle may rupture the upper arm. The contraction of the proximal muscles in the thigh as well as of the flexors and extensors can break the thigh or the lower leg.

Due to the extension [15] of the surface of the transversal section decreases, hence its ray $\mathrm{r}$ modified with $\Delta \mathrm{r}$, hence the $\mathrm{V}$ volume of the bone is modified:

$$
\frac{\Delta V}{V}=(1-2 \mu) \cdot \frac{F}{A \cdot E}
$$

where: $\mu-$ is the Poisson number, calculated according to the formula:

$$
\mu=\frac{d}{r} \cdot \frac{\Delta r}{\Delta d}
$$

After the extension, the bone appears like a deformed hyperboloid, which is given by the following formula:

$$
\varepsilon_{\mathrm{xx}} \cdot \mathrm{x}^{2}+\varepsilon_{\mathrm{yy}} \cdot \mathrm{y}^{2}+\varepsilon_{\mathrm{zz}} \cdot \mathrm{z}^{2}+2 \cdot \varepsilon_{\mathrm{xy}} \cdot \mathrm{xy}+2 \cdot \varepsilon_{\mathrm{xz}} \cdot \mathrm{xz}+2 \cdot \varepsilon_{\mathrm{yz}} \cdot \mathrm{yz}=\mathrm{k}
$$

where: $\mathrm{k}$ - a constant; $\varepsilon_{\mathrm{xx}}, \varepsilon_{\mathrm{yy}}, \varepsilon_{\mathrm{zz}}, \varepsilon_{\mathrm{xy}}, \varepsilon_{\mathrm{xz}}, \varepsilon_{\mathrm{yz}}$ - are the coefficient of the deformation tensor.

c) Torsion fractures result in a long, spiral fracture. In this case, the force is applied in opposite directions at both ends of the bone (e.g., torsional force near a fixed limb).

Torsional fractures occur when twisting a tubular bone at its two ends along its longitudinal axis, or twisting a fixed bone at the other end, e.g. if the torso is twisted with a fixed leg. In this case, tensile, compressive and displacement forces are applied. When the forces acting in the opposite direction overcome the elasticity and the rotational strength of the bone, a torsional fracture is created.

Torsion is usually accompanied by bending and compression. In the case of pure torsional fracture, the fracture line forms an angle of 45 degrees with the longitudinal axis of the bone. This angle increases with the application of bending force and decreases with the effect of compressive force. When twisting is accompanied by bending and compression, a diamond-shaped piece often breaks out of the bone. The oblique sides of the rhombus are caused by the force of torsion and the parallel sides by the force of bending and compression.

Torsion fractures can show a very pointed cone shape on the bone body, which can tear the skin from the inside. Bone parts tend to move.

d) In the case of compression fractures, the force acts along the longitudinal axis of the bone and presses the diaphysis into the proximal joint of the long tubular bones, resulting in a T or Y fracture line.

3) For complex mechanism fractures, the combination of the forces listed above causes the injury [18]. In this case, the fracture line also has mixed, transverse and oblique components, and usually even a larger butterfly-shaped mussel emerges from the oblique fracture.

4) Pathological fractures. If the fracture occurs as the result of a minimal, inadequate force trauma, then it is a pathological fracture. Any disease that alters the content of bone components (collagen, minerals) also reduces the solidity of the bone and predisposes the patient to pathological fractures: osteolysis, osteitis, tumors and systemic diseases of the bones. [19] Bone atrophy can be caused by defective functioning (inactivity), old age, tumor pressure, neurological disorders: softening of the brain, of the spinal cord, hollow spinal inflammation.

Osteitis: acute, chronic bone marrow inflammation, 
thinning osteoarthritis, fibrous osteitis, osteoarthritis, tuberculosis. Tumors: cystic tumor, chordoma, cancer, cyst, sarcoma. Systemic diseases of the bone such as rickets, osteomalacia, fracture of the bone, sinking, imperfect bone formation. In terms of fracture types, pathological fractures are similar to the types discussed above.

A distinction is made between single and multiple fractures: in the latter, a bone breaks at multiple locations, far apart and independently. Finally, multiple bones can break at once.

Almost $60 \%$ of adult fractures and $85 \%$ of childhood fractures are treated conservatively. [20] Conventionally, gypsum plaster, that is, a plaster splint or split circular gypsum is applied to the limb after the reposition. This allows for limb dilatation, which is necessarily due to any edema following fracture or repository manipulation. The skin of the limb should be protected with stockings and a soft felt-like material over the protruding bone parts. The extension is a semi-operative fracture treatment method, which consists of adjusting the fracture by longitudinal pull and direct pressure and replacing it with gypsum plaster in due course. Extension treatment involves long hospitalization with all its complications.

\section{The Treating of Fractures}

In treating a fracture, healing is only one of the goals. The other is good function, which is served by the complex rehabilitation program, which does not simply consist of exercise. These include active (isometric, isotonic, and isokinetic) muscle exercises, movement exercises (degrees of gravity cut-off and the so-called active movement against resistance), passive movements with properly designed electrical machines, current stimulation treatment, and other physiotherapy treatments to reduce pain and edema. [21]

Another purpose of functional therapy is to restore total joint movement amplitude and muscle strength, that is, restitution of function. [22] However, restoration of function affects the healing of fractures and soft tissues, as it is known that both the remodeling of bone beams, the bulking of fractures under load, and the formation of lateral collagen chains of the joint are due to function. Recognition of all these has led us to begin functional treatment as soon as possible. [23]

The fracture heals by a different mechanism in the standing diaphysis of the tubular bones than in the epiphysis of the bone ends. [24] The mechanism is also different, depending on how much micromobility between the fractures is allowed to fix the fracture.

\section{Conclusion}

The bones form the skeleton, which constitutes the passive part of the locomotory apparatus. The joints are the connection organs between the bones and they represent the place of the movements between the skeletal parts they get in contact with. The shape and structure of the joints are adapted to the function they fulfil - that of participating to the achievement of the movements and at the same time to resist to pressure and traction.

After the fracture, the healing process starts immediately, provided that the bone extremities have been reduced immediately and correctly in order for the bone to heal. The osteosynthesis consists in the reunion of the fragments of a fractured bone with the help of artificial structures: implants. According to the type and seriousness of the fracture, various metallic implants can be realized: plaques, screws, plaques with screws (associating the two types of implant) and broaches. The cast immobilization has a few risks, especially the one of an excessive compression of the muscles and nerves if the cast is too tight.

The natural process of bone healing is achieved in several phases. During the first phase, between the two bone extremities a blood clot is formed stopping the bleeding of the vessels destroyed by the fracture. In a second phase, a part of the while cells (macrophages) destroy the debris. Immediately after that, other cells (fibroblasts) form the matrix of the new bone tissue. During the third phase, between the two bone extremities of the fracture a new one appears (callus) erasing the fracture line. At the level of the callus a denser and thicker bone than the previous one is formed (the fourth phase). In the last phase (quite long), the bone reacquires an almost normal aspect. The time necessary for the consolidation of the fractures varies from one person to the other and depends on numerous factors. It takes 3 months for an adult to step well on the foot after a tibia fracture. In children, fractures heal much faster compared to the adults. The healing of a fracture depends on the diet as well. The more abundant the calcium and protein intake is, the better the results.

In the event of a fracture, the indication is: restoring fitness, providing care, relieving pain, eventually extending survival and achieving a decent terminal stage.

\section{References}

[1] J, Vincze, Medical Biophysics. Tom I., II., and III. [in Hungarian: Orvosi biofizika] NDP P. Budapest, 2018.

[2] Kogianni, G., Noble, B. S. (2007). The biology of osteocytes. Curr. Osteoporosis. Rep. 5: 81-86.

[3] Olsen, B. R., Reginato, A. M., Wang, W. (2000). Bone development. Annu. Rev. Cell Dev. Biol. 16: 191-220.

[4] Zaith, M. (2007). Skeletal remodelling in health and disease. Nat. Med. 13: 791-801.

[5] J, Vincze, The Structure and Biomechanics of the Human Skeleton. NDP P. Budapest, 2014.

[6] J, Vincze, The Microscopical Architectury of the Long Human’ Bones. NDP P. Budapest, 2017.

[7] Bonasso, P. C., Dossinger, M. S., et al. (2018). Review of beside surgeon-performed ultrasound in pediatric patients. J. Pediatric Surg. 11: 2279-2289. 
[8] Eckert K., Ackermann, O. (2015) Frakturdiagnostik. Radiologie 11: 992-999.

[9] Soles, G. L., Ferguson, T. A. (2012). Fragility fractures of the pelvis. 3: 222-228.

[10] Neviaser, R. J., Resch, H., Neviaser, A. S., Crosby, L. A. (2015). Proximal humeral fractures. Course Lect. 64: 203234.

[11] Molnar, T. F. (2017). Thoracic trauma. Thorac. Surg. Clin. 27: 13-23.

[12] Vachtaevanos L., Hayden, L., Desai, A. S., Dramis, A. (2014). Management of proximal fractures in adults. World J. Ortop. 5: 685-693.

[13] Katzer, C., Wasem, J., Eckert, K., Ackermann, O., Buchberger, B. (2016) Utility of diagnostic ultrasound in evaluating fracture in children. Pediatr. Emerg. Care. 6: 401-417.

[14] J, Vincze, Medical Biophysics. NDP P. Budapest, 2018.

[15] Vincze, J. (1991). The Relationship between the Primary and Secondary Architectonis of the Bones. Acta Biologica, Debrecina Suppl. 10: 47-54.

[16] J, Vincze, Biomechanics. NDP P., Budapest, 2016.
[17] J, Vincze, The Capital Chapters of Physics. NDP P., Budapest, 2018.

[18] Styrkarsdottir, U., Halldorson, B. V., Gretandottir, G. (2008) Multiple genetic loci for bone mineral density and fractures. N. Engl. J. Med. 358: 2355-2365.

[19] Kanis, J. A. (1994). Treatment of osteoporotic fracture. Lancet, 1: 27-33.

[20] Cheng, S. Y., Levy, A. R., Lefaivre K. A., et al. (2011). Geographic trends in incidence of hip fractures. Osteoporors Int. 10: 2575-2586.

[21] Smith, A. J. Lloyd, D. G., Wood, D. J. (2006). A kinematic and kinetic analysis of walking after knee artroplasty. $\mathrm{Cl}$. Biomechanics. 21: 379-387.

[22] Huysmans T., Haer, B., Van Andekerecke, R., Van der Slotem, J., Van der Perre, G. (2004) Three-dimensional mathematical reconstruction of the spinal share, based on active conture. J. of Biomechanisc, 37: 1793-1798.

[23] Lieberman, J. R., Dalnski, A. Einhorn, T. A.: (2002). The role of growth factors in the repair of bone. 84A: 1032-1044.

[24] Schmid, G. L., Lippmann, S. et al. (2017). The investigation of suspected fracture. Dtsch. Arztebl. Int. 45: 757-764. 